\title{
Frontières
}

\section{La mortalité par suicide au Québec}

\section{Richard Boyer et Danielle St-Laurent}

Volume 12, numéro 1, automne 1999

Suicides, générations et culture

URI : https://id.erudit.org/iderudit/1074508ar

DOI : https://doi.org/10.7202/1074508ar

Aller au sommaire du numéro

Éditeur(s)

Université du Québec à Montréal

ISSN

1180-3479 (imprimé)

1916-0976 (numérique)

Découvrir la revue

Citer cet article

Boyer, R. \& St-Laurent, D. (1999). La mortalité par suicide au Québec.

Frontières, 12(1), 50-53. https://doi.org/10.7202/1074508ar

\section{Résumé de l'article}

$\mathrm{Au}$ cours des deux dernières décennies, le taux de suicide québécois a presque doublé. Les hommes âgés de 35 à 39 ans sont actuellement les plus à risque de suicide. Le profil des suicides par génération suggère une augmentation des suicides non seulement chez les hommes les plus jeunes, mais aussi l'augmentation du risque suicidaire d'une génération à l'autre. Les taux de suicide chez les jeunes continuent de progresser de façon systématique et la suicidalité des générations du baby-boom semble se maintenir avec

l'avancement en âge. Si les jeunes générations masculines maintiennent en vieillissant l'écart par rapport aux autres générations du baby-boom, elles pourraient présenter des taux de suicide très élevés au fur et à mesure de leur avancement en âge. 


\section{Résumé}

Au cours des deux dernières décennies, le taux de suicide québécois a presque doublé. Les hommes âgés de 35 à 39 ans sont actuellement les plus à risque de suicide. Le profil des suicides par génération suggère une augmentation des suicides non seulement chez les hommes les plus jeunes, mais aussi l'augmentation du risque suicidaire d'une génération à l'autre. Les taux de suicide chez les jeunes continuent de progresser de façon systématique et la suicidalité des générations du baby-boom semble se maintenir avec l'avancement en âge. Si les jeunes générations masculines maintiennent en vieillissant l'écart par rapport aux autres générations du babyboom, elles pourraient présenter des taux de suicide très élevés au fur et à mesure de leur avancement en âge.

Mots clés: suicide - Québec - hommes générations - statistiques

\section{Abstract \\ Over the past two decades the suicide rate nearly doubled in Québec. Men aged 35 to 39 are at the greatest risk for suicide. The profile of suicides per gene- ration suggests not only an increase in suicides among the youngest men, but also the increase in the risk of suicide from one generation to another. The sui- cide rates among young people continue to progress systematically and the suici- dality of baby-boom generations seems to remain steady with advancement in age. If younger male generations, while aging, maintain the difference compared with other baby-boom generations, they could show very high suicide rates as they age.}

Key words: suicide - Québec - men generations - statistics

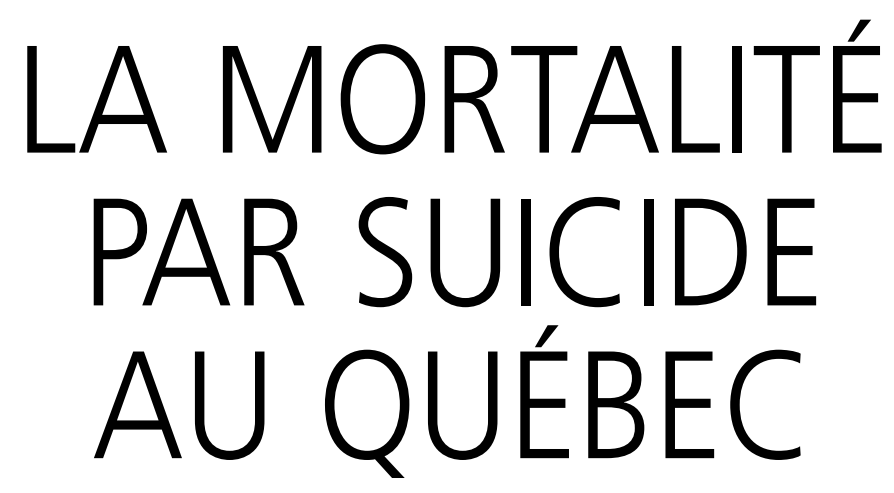

Richard Boyer,

directeur scientifique, Institut universitaire de gérontologie sociale du Québec

et chercheur au Centre de recherche Fernand-Séguin.

Danielle St-Laurent,

épidémiologiste, Institut national de santé publique du Québec.

L'objectif de cet article est de décrire l'évolution du taux de suicide au Québec en fonction de l'âge et des générations. Pour obtenir un tableau adéquat de la situation québécoise, il est essentiel de distinguer l'incidence $\mathrm{du}$ suicide chez les hommes et les femmes en fonction de l'âge. Cette étude débute en décrivant les taux de suicide québécois dans son ensemble pour ensuite se concentrer sur les distributions en fonction du sexe et de l'âge pour les années 1971 à 1996. Puisqu'il faut tenir compte des variations historiques sensibles dans la structure d'âge de la population québécoise pouvant fausser l'interprétation de l'évolution de ce phénomène, il est nécessaire d'utiliser des taux standardisés. Ainsi, les populations québécoises de chaque année sont comparées après avoir appliqué une standardisation directe qui consiste à calculer le taux de mortalité par âge et par sexe sur la base de la structure de la population du Québec de 1991.

L'analyse des taux de mortalité par suicide par groupe d'âge est informative en soi; il nous faut aussi postuler que les jeunes, les adultes et les aînés diffèrent peu en termes culturel ou comportemental d'une génération à l'autre, alors que les normes et les exigences de la société québécoise vis-à-vis les hommes et les femmes des différents groupes d'âge ont grandement évoluées. Pour mieux tenir compte de ces questions, il est nécessaire d'analyser l'évolution des taux de mortalité par suicide dans une perspective longitudinale; cela permet de suivre les variations de comportement d'un groupe de générations à un autre, à un âge donné1. Nous résumerons les analyses menées par St-Laurent pour les années 1971 à $1995^{2}$. Par la suite, nous comparerons le profil du Québec avec le Canada et les États-Unis.

\section{TAUX DE SUICIDE POUR L'ENSEMBLE DE LA POPULATION (1976 À 1996)}

$\mathrm{Au}$ cours des deux dernières décennies, le phénomène du suicide a pris beaucoup d'ampleur au Québec. Le taux de suicide québécois a presque doublé, passant de 12,9 pour 100,000 personnes en 1976 à 19,6 pour 100,000 personnes en 1996, soit une augmentation de $52 \%$. Derrière cette statistique de 1996 se cache la souffrance psychologique de 1463 personnes, soit 1136 hommes et 327 femmes.

La première caractéristique de la population québécoise est qu'il y a un écart du taux comparatif de mortalité entre les hommes et les femmes et que cet écart s'accroît constamment (Figure 1). Entre 1976 et 1996, le taux a augmenté de 2,7 points (augmentation de $47 \%$ ) chez les femmes tandis que, chez les hommes, cette progression était de 15,0 points (augmentation de $94 \%$ ). 
Il en résulte qu'en 1996, la mortalité par suicide est de 31,3 pour 100000 hommes et de 7,9 pour 100000 femmes.

Si l'on regarde de plus près l'évolution année après année, la période de 1976 à 1983 est marquée par une progression beaucoup plus rapide chez les hommes que chez les femmes. Chez les femmes, cette période présente une tendance à la décroissance qui s'interrompt en 1991, pour ensuite augmenter de $36 \%$ entre 1991 et 1996, le taux passant de 5,8 à 7,9 pour 100000 personnes. Chez les hommes, on n'a pas constaté le net recul observé chez les femmes après 1983. Le taux de suicide des hommes a évolué en dents-de-scie entre 1983 et 1989 avec une légère tendance à la baisse. Après 1989, la tendance se renverse et le taux de mortalité par suicide chez les hommes augmente de $19 \%$, passant de 26,1 à 31,0 en 1996.

Figure 1

Taux comparatifs de mortalité par suicide pour 100000 personnes, Quebec, 1976-1996

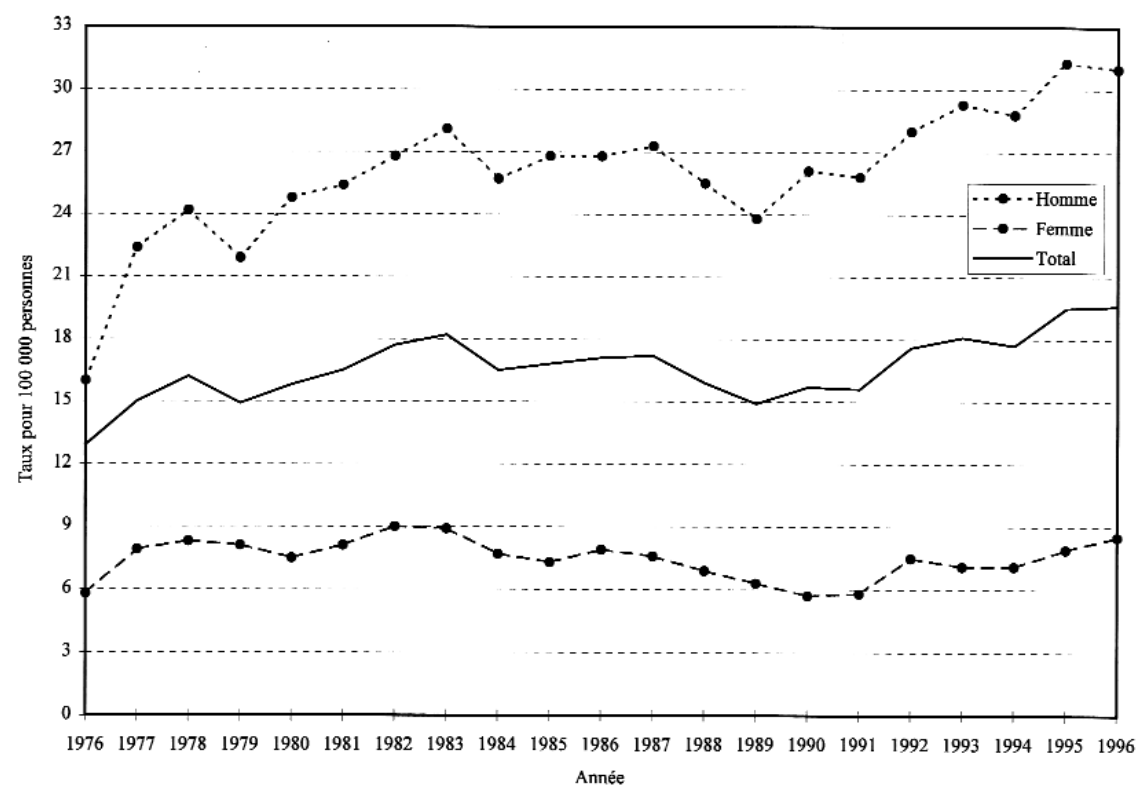

Sources : Fichier des decès, ministère de la Santé et des Services sociaux

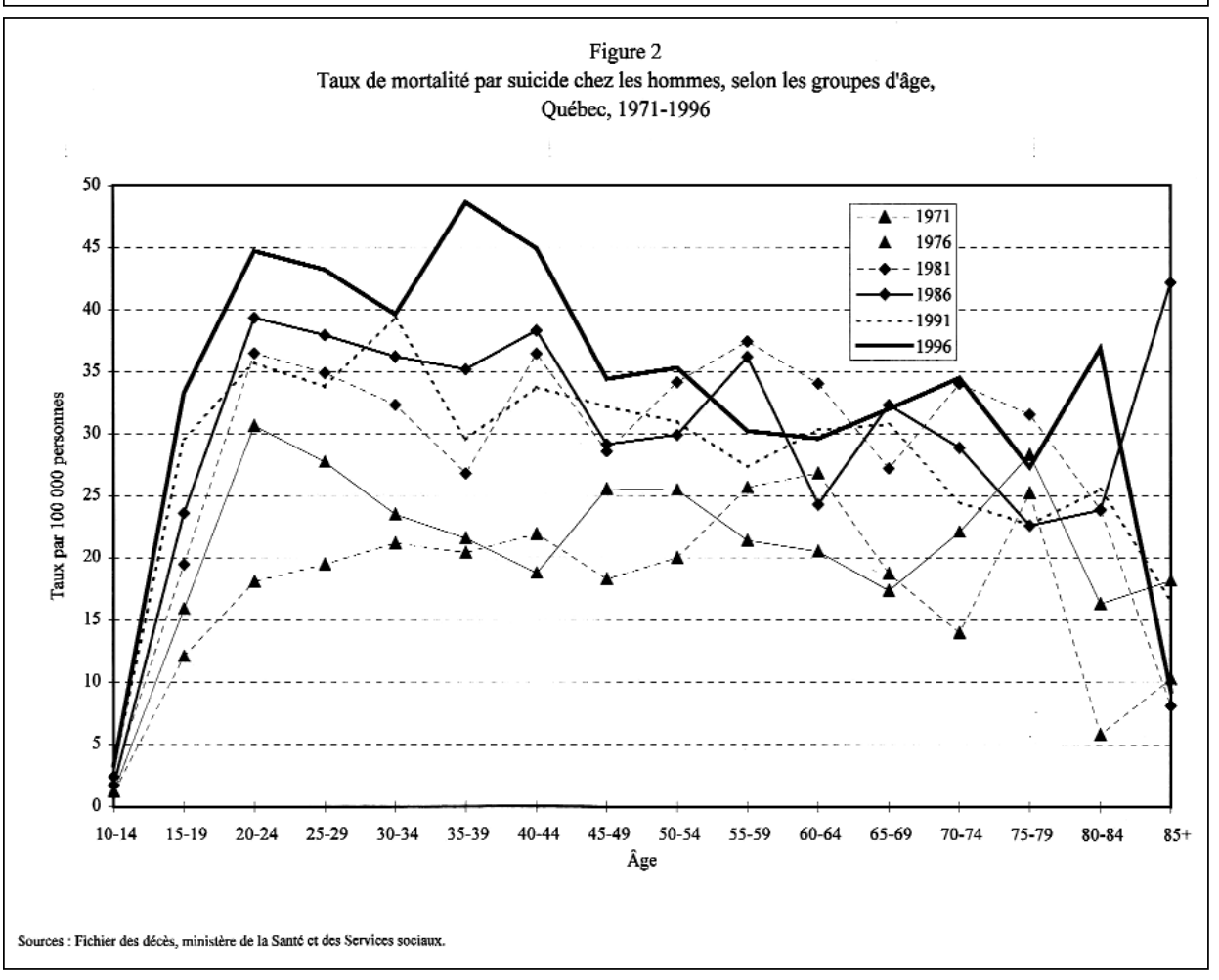

\section{ÉVOLUTION SELON L'ÂGE}

Les figures 2 et 3 présentent l'évolution des taux de suicide selon l'âge (groupes d'âge quinquennaux) chez les hommes et les femmes respectivement. Chez les hommes, on constate que la variation des taux de suicide dans le temps est nettement plus importante chez ceux de moins de 50 ans comparée aux hommes âgés entre de 50 et 79 ans. Les fortes variations observées chez les aînés (80 ans et plus) s'expliquent par un nombre relativement plus faible de suicides dans ce groupe d'âge. Au fur et à mesure que les années avancent, on constate clairement un déplacement des plus hauts taux de suicide dans les populations d'hommes les plus jeunes et tandis que les taux de mortalité par suicide chez les hommes de 55 et plus ont beaucoup moins fluctué. Le profil de 1996 révèle que les hommes de 35 à 39 ans sont plus à risque de suicide. Chez les femmes, les taux de mortalité par suicide selon l'âge sont beaucoup plus stables. Ici, pour chaque année, la mortalité par suicide s'accroît jusqu'à 44 ans pour ensuite connaître de fortes variations pour les groupes d'âge subséquents. Toutefois, la courbe de 1996 se caractérise par des taux décroissants après 49 ans.

\section{ANALYSE DES GÉNÉRATIONS}

Les générations étudiées sont les suivantes: 1906-1911 et 1971-1976. Cependant, faute de données valides pour les générations les plus vieilles et d'absence de données pour les générations les plus jeunes, la brièveté de la période couverte par l'analyse ne permet pas d'analyser la «suicidalité» de toutes les générations, c'est-à-dire la propension au suicide sur l'ensemble de la vie d'une génération, mais seulement sur une tranche variable de vie de chacune.

Les générations masculines sont présentées à l'aide de la Figure 4. En premier lieu, on constate que les courbes des générations formées par les personnes nées à partir de 1936 se distinguent des précédentes par une mortalité toujours plus élevée jusqu'à 40 ans. Le taux de suicide des hommes de 40-44 ans de la génération de 1941-1946 est plus élevé $(34,8)$ que celui de la génération de 1936-1941 (32,6). Aux âges plus avancés, les courbes des générations formées par les personnes nées avant la cohorte de 1936-1941 montrent plus de chevauchements, témoignant ainsi d'une moindre régularité dans les tendances. La hausse des taux de suicide d'une génération à l'autre (à partir de la cohorte de 1921-1926) chez les groupes d'âge de 15 à 49 ans est très nette, alors que 
chez les groupes d'âge qui suivent, on observe une tendance à la baisse.

Le profil par génération suggère une augmentation des suicides non seulement chez les hommes les plus jeunes, mais aussi l'augmentation du risque suicidaire d'une génération à l'autre. Par exemple, les générations formées par les personnes nées entre 1941 et 1946 ont un taux de suicide de 22,3 pour 100000 personnes à l'âge de 20-24 ans et celles formées par les personnes nées en 19661971, de 45,4. Les générations formées par les personnes nées entre 1931 et 1936 ont un taux de suicide de 21,3 à l'âge de 30-34 ans tandis que celles de 1956-1961, de 42,8 au même âge. Si la génération dite du baby-boom (19461966) manifeste des taux de suicide supérieurs par rapport aux générations antérieures, ce phénomène existe aussi chez les générations précédentes et les différences intergénérationnelles semblent encore plus marquées avant le baby-boom. La Figure 4 suggère que si les tendances observées se poursuivent avec l'avancement en âge des générations masculines du baby-boom et celles qui le suivent, il y aura un accroissement du nombre de suicides avec l'âge au cours des prochaines décennies.

Selon la Figure 5, la situation des femmes est beaucoup plus complexe. Chez les plus jeunes, soit celles nées après 1956, le risque suicidaire varie peu d'une

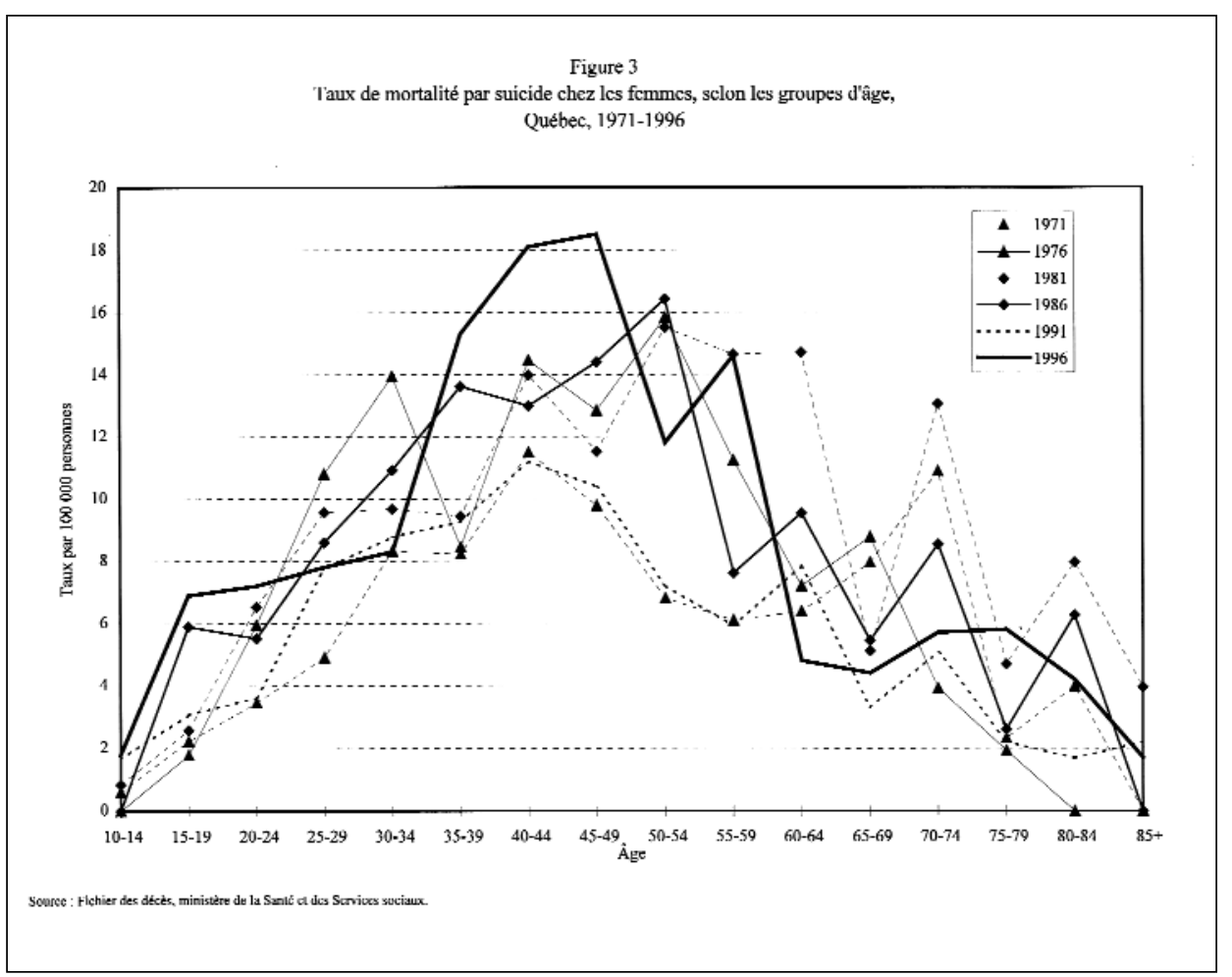

génération à l'autre. Nous pouvons cependant observer le début d'un effet de cohorte. Par exemple, la génération de 1956-1961 présente un taux de suicide de 2,8 à l'âge de 15-19 ans et celles nées entre 1971 et 1976 (une seule mesure), un taux de 7,0. Pour les générations nées avant 1956, les profils de suicide selon l'âge sont beaucoup plus difficiles à interpréter. On observe des taux élevés entre 40 et 54 ans chez les générations nées entre 1921 et 1936 . Les femmes de toutes ces générations semblent présenter un comportement beaucoup plus homogène que les hommes et on peut penser que le risque suicidaire a peu changé. 

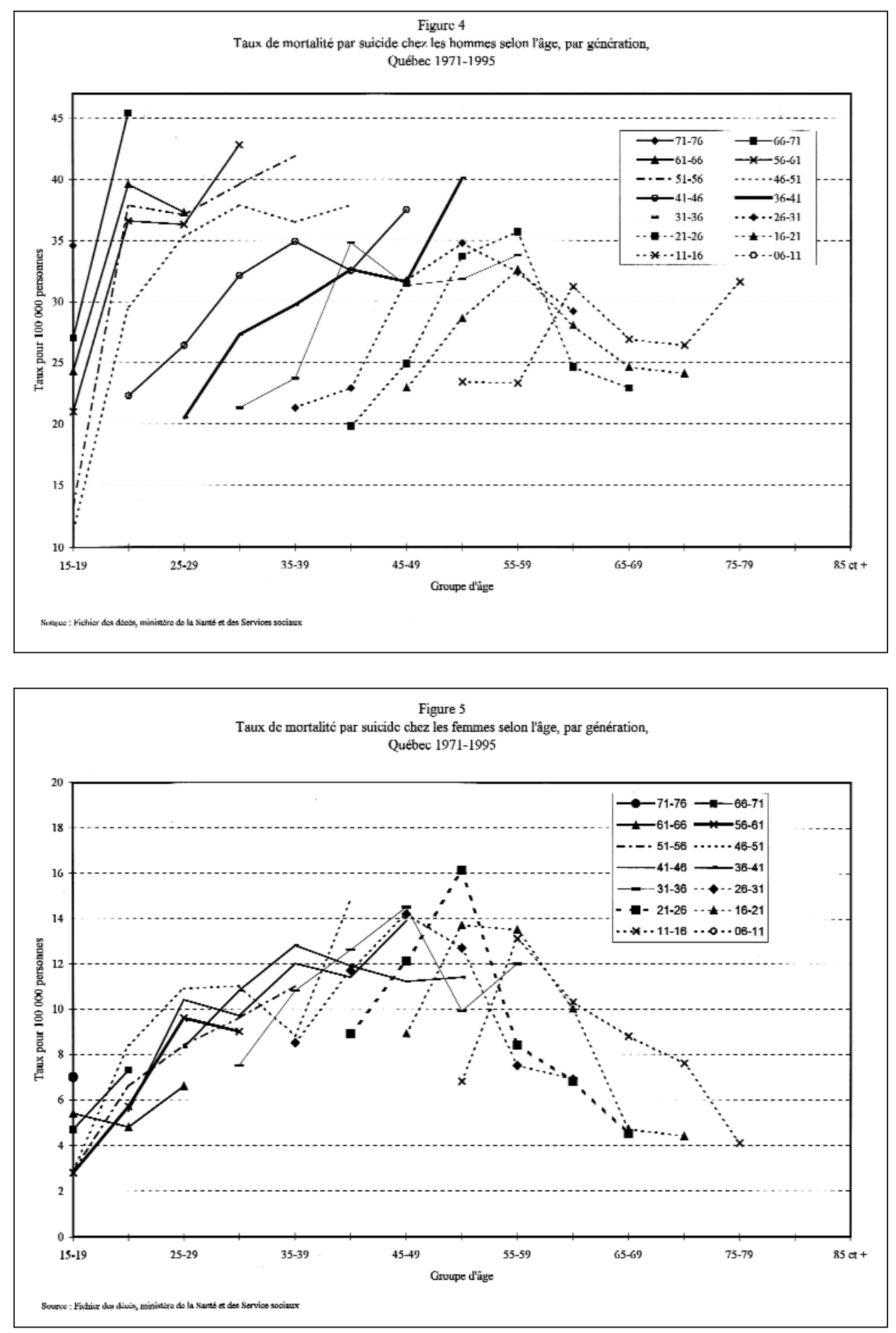

\section{COMPARAISON}

\section{QUÉBEC - CANADA}

Si la distribution des suicides selon le sexe au Canada et au Québec montre que l'incidence du suicide est beaucoup plus grande chez les hommes, le Québec se démarque du Canada par une constante progression de la sur-représentation des hommes depuis 20 ans. La distribution québécoise des suicides en fonction de l'âge est particulière et ne suit pas le profil observé au Canada et aux États-Unis. Tant dans l'un que l'autre pays, on observe depuis une vingtaine d'années une augmentation du sonnes en 1991), dépasse ceux de l'Italie $(2,6)$, de l'Espagne $(2,6)$, des Pays-Bas $(4,0)$, de l'Angleterre $(4,3)$ du Japon $(5,2)$, des États-Unis $(6,6)$, de la Belgique $(7,4)$, de l'Australie $(7,5)$, du Danemark $(8,1)$ et de la France $(8,2)$. Pour les femmes, la comparaison internationale est moins sombre tant pour le taux de mortalité par suicide que pour le nombre potentiel d'années de vie perdues. En 1991, le taux de suicide des femmes était de 2,0 pour 100000 personnes, comparativement à 3,4 en Finlande, 3,1 au Danemark, 2,9 en Suède, 2,7 en Suisse et en France et de 2,8 en Belgique 5 .

\section{CONCLUSION}

La très forte augmentation du taux de suicide au Québec est marquée par des tendances démographiques lourdes. Les hommes contribuent le plus à cette augmentation. On a constaté un net déplacement des plus hauts taux de suicides chez les populations d'hommes les plus jeunes alors que les taux de mortalité par suicide chez les hommes de 55 ans et plus ont beaucoup moins fluctué. Les hommes de 35 à 39 ans sont actuellement les plus à risque de suicide. L'approche longitudinale utilisée ici a aussi permis de montrer que la suicidalité des hommes a commencé à progresser avant l'arrivée des générations du babyboom et que ce phénomène semble se confirmer avec celle-ci. Depuis, le taux de suicide chez les jeunes continue de progresser de façon systématique de génération en génération alors que la suicidalité des générations du babyboom semble se maintenir avec l'avancement en âge. Si ces générations masculines de 1966 à 1976 maintiennent en vieillissant l'écart par rapport aux autres générations du baby-boom, elles pourraient présenter des taux de suicide très élevés au fur et à mesure de leur avancement en âge. Dans un tel cas, la hausse de la mortalité par suicide au Québec se poursuivra.

\section{Notes}

1 P. SURRAULT, «Variation du suicide en France», Population, vol. 4-5, 1995, p. 983.

2 H. GAUTHIER et al., Statistiques sociales, Bureau de la statistique du Québec, volume II, 1998, p. 68.

3 D. LESTER et A. A. LEENAARS, «Suicide in Canada and United State: A Societal Comparison", dans Suicide in Canada, A. A.LEENAARS et al. (dir. publ.), University of Toronto Press, 1998, p. 109.

4 R. BOYER, G. LÉGARÉ, D. ST-LAURENT, et M. PRÉVILLE, «Epidemiology of Suicide, Parasuicide, and Suicide Ideation in Quebec», dans Suicide in Canada, ibid, p. 67.

5 Ibid. 